\title{
Cross - resistance between antiseptic agents and antimicrobial agents
}

Keywords: antiseptic agents, antimicrobial agents, crossresistance, paucity of evidence, environmental contamination, triclosan

\section{Introduction}

Antimicrobial resistance is evolving into a huge public health challenge. The increasing burden of infectious diseases necessitated the increased use of antibiotics which in turn contributed to the growing burden of antimicrobial resistance. ${ }^{1}$ The global report on antimicrobial surveillance by WHO in 2014 identified major gaps in the knowledge along with paucity of evidence for quantification of the burden of antimicrobial resistance accurately. ${ }^{2}$ It highlighted the complex interaction between antimicrobial resistance and various environmental and human health factors. One of these factors is interaction between the use of antiseptics and antibiotics. One of the results of such interaction is the development of resistance to antibiotic agents.

Commonly, antiseptic agents are used as preventive agents while antimicrobial agents are used for therapeutic purposes. Crossresistance of antiseptic agents and antimicrobial agents is not thoroughly studied. Theoretically, it is possible since antiseptic agents sometimes act at the same receptors or pathways as the antimicrobial agents to curtail the infections. Due to this, exposure to antiseptic agents can trigger mutations in the receptors or the pathways which can result in the development of antimicrobial resistance. The term "cross- resistance" in the field of antimicrobial resistance is poorly defined. Many authors use the term "cross- resistance" to indicate the development of resistance to different classes of antimicrobials such as $\beta$ - lactams, aminoglycosides, polymyxins etc. ${ }^{3-5}$ It is often used in the same context as multi- drug resistance. For this paper, cross- resistance specifically refers to the development of resistance to both antiseptic agents and antimicrobial agents. Very few studies are available on the cross- resistance between antiseptic agents and antimicrobial agents. Wand et al found that invitro exposure of Klebsiella pneumoniae cultures to chlorhexidine resulted in the development of colistin resistance. ${ }^{6}$ They detected specific mutations in the $P h o P / Q$ following the exposure to chlorhexidine that resulted in the development of resistance to both chlorhexidine and colistin.

Several studies investigated the development of resistance to various antimicrobials following exposure to triclosan. Cary et al., ${ }^{7}$ found that chronic exposure to triclosan is associated with increased resistance to various antibiotics such as rifampicin, ciprofloxacin, gentamycin etc. Werckenthin ${ }^{4}$ studied the resistance genes to various antimicrobial chemicals which include antiseptics and antibiotics in indoor dust microbiome. ${ }^{8}$ They found that there is significant correlation between antimicrobial resistance genes such as $\beta$ - lactamases, aminoglycoside acetyl transferases and antiseptic resistance genes for several antibiotics like triclosan, triclocarban and butylparaben. Another significant point to consider is the environmental contamination with antiseptics. Studies report that antiseptics such as chlorhexidine and benzalkonium chloride are frequently detected in the sewage sludge
Special Issue - 2018

\author{
Lavanya Gudapuri \\ Division of Clinical Microbiology, Rush University Medical \\ Center, USA
}

Correspondence: Lavanya Gudapuri, Division of Clinical Microbiology, Rush University Medical Center, 100 S Buffalo Grove Road Apt I I3, USA, Tel 2243180076,

Email lavanya.gudapuri@gmail.com

Received: July 30, 2017 | Published: September 24, 2018

and wastewater at high levels (up to $19 \mu \mathrm{g} / \mathrm{ml}$ ). ${ }^{9}$ The global market for antiseptics and disinfectants accounted for USD 5.55 billion in 2015 and estimated to reach USD 8.1 billion by $2021 .{ }^{10}$ This shows the increase in the use of antiseptics worldwide which ultimately end up in the sewage and waste water contaminating the environment. Presence of high level antiseptics can trigger mutations in the bacteria facilitating the development of multidrug resistant strains.

Gradea et al. studied the effect of exposure to quaternary ammonium compounds (commonly used antiseptic agents) on several strains of bacteria. ${ }^{11}$ They reported that $88 \%$ of the isolates developed decreased susceptibility to other antiseptic agents such as chlorhexidine, triclosan, hexachlorophene etc. They also found resistance to ampicillin, sulfamethoxazole, cefotaxime etc. in the same isolates. However, further research is needed to determine if there is an association between the presence of antiseptic agents and development of antimicrobial resistance. Since the is predicted increase in the antiseptic usage in the recent future, the implications of such usage should be identified. Measures to curtail any health challenges posed by the increased usage of the antiseptics should be designed. A step towards such progress would be the ban of triclosan by the FDA. ${ }^{12}$ The FDA banned the use of triclosan in the US stating that it could cause health risks such as antimicrobial resistance. While banning of antiseptics would be hazardous, regulating the antiseptic consumption can be an achievable goal considering the environmental contamination by the antiseptics.

\section{Conclusion}

Further evidence is needed to support the theory of crossresistance between the antiseptics and antimicrobials. The interaction between the development of resistance and exposure to antiseptic agents should be evaluated in depth for any contributing factors. Such knowledge will help in designing public health interventions that are effective in preventing future challenges of cross resistance.

\section{Acknowledgements}

None. 


\section{Conflict of interest}

Author declares that there is no conflict of interest.

\section{References}

1. Bejarano MT. The Global Public Health Threat of ABR Preserving existing and future antibiotics. 2017.

2. World Health Organization. Global Action Plan on Antimicrobial Resistance. Switzerland: Geneva; 2015.

3. Gilbert P, Allison DG, McBain AJ. Biofilms in vitro and in vivo: do singular mechanisms imply cross- resistance? Symp Ser Soc Appl Microbiol. 2002;31:98S-110S.

4. Werckenthin C, Böttner A, Hafez HM, et al. Cross- resistance between antimicrobial agents used in veterinary medicine: molecular background and practical consequences for susceptibility testing. Berl Munch Tierarztl Wochenschr. 2005;118(11-12):471-480.

5. Gnanadhas DP, Marathe SA, Chakravortty D. Biocides- resistance, crossresistance mechanisms and assessment. Expert Opin Investig Drugs. 2003;22(2):191-206.

6. Wand ME, Bock LJ, Bonney LC, et al. Mechanisms of Increased Resistance to Chlorhexidine and Cross-Resistance to Colistin following Exposure of Klebsiella pneumoniae Clinical Isolates to Chlorhexidine. Antimicrob Agents Chemother. 2016;61(1).
7. Carey DE, Zitomer DH, Kappell AD, et al. Chronic exposure to triclosan sustains microbial community shifts and alters antibiotic resistance gene levels in anaerobic digesters. Environ Sci Process Impacts. 2016;18(8):1060-1067.

8. Erica Hartmann M, Roxana Hickey, Tiffany Hsu, et al. Antimicrobial Chemicals Are Associated with Elevated Antibiotic Resistance Genes in the Indoor Dust Microbiome. Environ Sci Technol. 2016;50(18):98079815 .

9. Östman M, Lindberg RH, Fick J, et al. Screening of biocides, metals and antibiotics in Swedish sewage sludge and wastewater. Water Res. 2017; 115:318-328.

10. Sarasota FL. Global Antiseptics and Disinfectants Market will reach USD 8.10 Billion by 2021: Zion Market Research. 2017.

11. Gadea R, Fernández Fuentes MÁ, Pérez Pulido R, et al. Effects of exposure to quaternary-ammonium-based biocides on antimicrobial susceptibility and tolerance to physical stresses in bacteria from organic foods. Food Microbiol. 2017;63:58-71.

12. US Food and Drug Administration. FDA issues final rule on safety and effectiveness of antibacterial soaps: Rule removes triclosan and triclocarban from over- the- counter antibacterial hand and body washes. Maryland, USA. 2016. 